\title{
INTERFERON GAMMA AND INTERLEUKIN-10 LEVELS IN PBMC OF ACTIVE AND LATENT TUBERCULOSIS PATIENTS AS WELL AS HEALTHY INDIVIDUALS
}

\author{
I G.A. Wiradari Tedja ${ }^{1}$, Jusak Nugraha ${ }^{1}$, Betty Agustina Tambunan ${ }^{1}$, Fransisca Sri Oetami ${ }^{2}$ \\ ${ }^{1}$ Department of Clinical Pathology, Faculty of Medicine, Airlangga University/Dr. Soetmo Hospital, Surabaya, Indonesia. \\ E-mail: iu_radari@yahoo.com ${ }^{2}$ Indonesian Health Research and Development Agency
}

\section{ABSTRACT}

Tuberculosis (TB), an infectious disease caused by a Mycobacterium tuberculosis, is still a health problem in Indonesia, and the world. One of the failures to control the TB epidemic is due to the lack of effective vaccines available today. Protective immune responses to $M$.tuberculosis are dominated by cellular immunity and less by humoral immunity. IFN- $\gamma$, and IL-10 play a role in the protection of against M.tuberculosis, and the pathogenesis of TB. Fusion antigen ESAT-6-CFP-10 has a strong antigenicity to T cells and stimulates specific cellular immune responses, thereby providing benefit in immune responses that are protective against M.tuberculosis infection. The aimed of this study was to know the difference betwen IFN- $\gamma$, and IL-10 levels on PBMC culture of active TB, latent TB, and healthy people after ESAT-6-CFP-10 fusion antigen stimulation. This study used an in vitro of quasi experimental design in PBMC cultures of active TB, latent TB, and healthy people groups stimulated by ESAT-6-CFP-10 antigen fusion Mycobacterium tuberculosis. IFN- $\gamma$, and IL-10 levels were measured by ELISA method. The results were analyzed by one-way ANOVA. The mean levels of IFN- $\gamma$ post-stimulation of ESAT-6-CFP-10 fusion antigens did not differ $(p=0.359)$ in the active pulmonary TB group (0.07 - 2114), latent TB (6.84 - 1381) and healthy people 1.88 - 1807.70), as well as the mean levels of IL-10 $(p=0.712)$ in the active pulmonary TB $(16.70-328.80)$, latent TB $(29.70-323.60)$ and healthy people (31.30 958). There were no significant differences in levels of IFN- $\gamma$ and IL-10 in active TB, latent TB, and healthy people after stimulation by fusion antigen ESAT-6-CFP-10.

Keywords: Tuberculosis, interferon gamma, interleukin-10, fusion antigen ESAT-6-CFP-10 after-transfusion.

\section{INTRODUCTION}

Tuberculosis (TB) is an infectious disease caused by Mycobacterium tuberculosis bacteria. The disease is still considered as a global concern since one-third of the world's population has been infected with these bacteria. TB attacked 9.6 million people and led to deaths of about 1.2 million in 2014. India, Indonesia, and China are countries with a high infected population, respectively $23 \%, 10 \%$ and $10 \%$. In 2014 the incidence of TB in Indonesia even reached 399 out of 100,000 population with a mortality rate of 41 from 100,000 population. ${ }^{1}$

TB patients with positive acid-fast bacteria smear are a source of TB infection, but only $5 \%-10 \%$ of exposed individuals will suffer from active TB, while others will suffer from latent $\mathrm{TB} .{ }^{1-4}$ Individuals with latent TB have a high risk of becoming active TB for life. Besides, TB is still considered as a global challen- ging problem to be controlled, including in diagnosing and treating individuals with latent TB who can act as reservoirs for new TB cases, especially in TB endemic areas. $^{5}$

The failure of epidemic TB control, moreover, is also due to the ineffectiveness of available vaccines, the resistance of M.tuberculosis to anti-tuberculosis drugs, and the lack of sensitivity and rapid diagnosis of TB. $^{6}$ Global control, and eradication of tuberculosis require the antigenic identification of M.tuberculosis that is useful for the specific diagnosis and development of effective vaccines for the protection of all tuberculosis. The development of a TB vaccine, thus, is expected to look for a gene product capable of providing a protective immune response to M.tuberculosis by identifying Mycobacterium antigens that can be recognized by T cells. ${ }^{7}$ The use of the Bacillus CalmetteGuerin (BCG) M.bovis vaccine, for instance, has curen- 
tly and widely been used as a protection against human TB in the world. Nevertheless, it remains controversial due to its failure to protect TB in adults, especially in endemic areas of TB. ${ }^{8}$

Furthermore, M.tuberculosis is metabolically active in producing ESAT-6, and CFP-10 secretion proteins. ESAT-6, and CFP-10, therefore, can potentially be used as antigens in the diagnosis of tuberculosis as well as in the production of an effective vaccine. ${ }^{9}$ M.tuberculosis which is viable even can stimulate a protective immune response, while the dead germs cannot.

Consequently, most of the proteins secreted by living M.tuberculosis (metabolism and active replication) may become essential antigens affecting the host's cells infected with T cells. ${ }^{10}$ ESAT-6, and CFP-10 fusion antigens, for instance, have a strong antigenicity to $T$ cells, and also can stimulate specific cellular immune responses very strongly as well as release IFN- $\gamma$ highly in tuberculosis patients, therefore, these antigens are known to provide benefits in protective immune response against M.tuberculosis infection. ${ }^{7}$

Besides, the protective immune response to M.tuberculosis is dominated by cellular, and limited humoral immunity. Both of these responses play a role in preventing and curing the disease although they are not at the same level. ${ }^{11}$ Interferon-gamma (IFN- $\gamma)$ is a major cytokine that plays a role in promoting a critical immune response to M.tuberculosis infection. Several types of research even have demonstrated the production of IFN- $\gamma$ in TB control. For instance, Cooper et al, and Flynn et al. ${ }^{14}$ revealed that animals with severe deficiencies in IFN- $\gamma$ had severe M.tuberculosis infection. ${ }^{12,13}$ It means that individuals with a deficiency in IFN- $\gamma$ are particularly susceptible to M.tuberculosis infection. ${ }^{14}$

Besides this, some types of research on humans and infected animals also found that $\mathrm{T} \mathrm{CD4}^{+}$, interleukin-12 (IL-12), gamma interferon (IFN- $\gamma$ ), and alpha necrotizing factor tumor (TNF- $\alpha$ ) cells are the basis of M.tuberculosis infection control. Nevertheless, it is still incomprehensible to explain why some people may be protected from M.tuberculosis infection, while in others it develops into a disease. ${ }^{6}$

Moreover, antigens triggering a robust IFN- $\gamma$ response can be considered as a candidate for the development of the tuberculosis vaccine. Meanwhile, antigens triggering Interleukin-10 (IL-10) are still debatable debatable in the production of the tuberculosis vaccine. Interleukin-10 is known to suppress the production of protective cytokines, i.e. IFN- $\gamma$, TNF- $\alpha$,
IL-1 and IL-12. ${ }^{15}$ Interleukin-10 can also inhibit the phagocytosis, and elimination of M.tuberculosis by limiting the production of reactive oxygen, and nitrogen intermediate mediated by IFN- $\gamma$, as well as inhibit phagosome maturation, thus facilitating the survival, and development of M.tuberculosis bacilli. ${ }^{16}$

Interleukin-10 is produced by Th2 cells, namely subsets of $\mathrm{T} \mathrm{CD}^{+}$cells including Th1 and Th17, B cells, neutrophils, and macrophages, as well as some subsets of dendritic cells. Increased IL-10 levels then support the life of Mycobacterium in the host's body. Similarly, a research conducted on a rat with less IL-10 showed an increase in its immunity against mycobacteria. The increased IL-10 levels in TB patients actually can also be associated with an increased incidence of TB and inefficiency of BCG vaccine. ${ }^{14}$

As a result, the high rate of TB incidence due to the lack of optimal control of M.tuberculosis infection triggers further researches to be focused more on an effective vaccine, stimulating a protective immune cell response. Therefore, this research aimed to analyze in vitro cellular immune responses, especially IFN- $\gamma$ and IL-10, of patients with active and latent TB, as well as healthy individuals after the stimulation of ESAT- 6 and CFP-10 fusion antigens.

\section{METHODS}

This research was conducted in the inpatient and outpatient installations of Karang Tembok Lung Hospital for six months (from October 2016 to March 2017). All participants who had signed consent forms were over 18 years old. Those research participants then were classified into three groups, each of which consisted of sixteen individuals. The first group consisted of those individuals diagnosed with active tuberculosis (no extrapulmonary TB) by pulmonologists. The second group consisted of nurses diagnosed with latent TB since they were at risk of contracting tuberculosis due to their duty for more than six months. And the last group consisted of healthy individuals who had negative sputum smear results, normal X-Ray photos, and negative tuberculin test. Those healthy individuals classified into the third group also had never been diagnosed with pulmonary TB or extrapulmonary TB, and never suffered from respiratory infections. This research then has been approved by the Research Ethics Commission of the Faculty of Medicine, Airlangga University. 
In addition, this research was considered as an in vitro quasi-experimental study with nonrandomized post-test only controlled group design. This research was focused on the Peripheral Blood Mononuclear Cell Culture (PBMC) of the three sample groups induced by M.tuberculosis antigens, namely ESAT- 6 and CFP- 10 . The specimen used was $5 \mathrm{~mL}$ of blood collected in a lithium heparin tube, and then PBMC isolation was performed. The density of lymphocyte cells $/ \mathrm{mL}$ used was made up of one million cells in $1 \mathrm{~mL}$ of culture medium containing RPMI 1640 to which 10\% Foetal Calf Serum and 1\% antibiotic/antimycotic (Penstrep + Amphotericin B).

Next, to all PBMC suspensions, ESAT-6, and CFP-10 fusion antigens were added, and then incubated for 5 days at a temperature of $37^{\circ} \mathrm{C}$ together with $5 \% \mathrm{CO}$. After the five days, the incubated PMBC suspensions were centrifuged at $1,000 \mathrm{~g}$ for 15 minutes. $100 \mu \mathrm{L}$ of their supernatants were then taken, and put into $1.5 \mathrm{~mL}$ Eppendorf tubes. After- simultaneously. Results obtained were in the forms of Standard Deviation (SD), and Coefficient of Variation (CV). Before the research, optimization was performed first, which aimed to determine the levels of ESAT-6 and CFP-10 fusion antigens used to stimulate the optimal cytokine secretion of IFN- $\gamma$, and IL-10. The dose of ESAT-6, and CFP-10 fusion antigens used in this research was $5 \mu \mathrm{g} / \mathrm{mL}$ since optimal lymphocyte proliferation was obtained at the dose.

Moreover, this research was conducted for six months. The number of subjects in this research was forty-eight individuals. Those subjects then were classified into three groups, namely active TB, latent TB, and healthy individuals. Each of the groups consisted of sixteen individuals (33.33\%).

Based on the data, the number of male patients in the active TB group was twelve individuals (75\%), higher than the females. Meanwhile, in the latent TB group, the number of female patients was higher than males, as many as 11 individuals (68.8\%).

Table 1. Characteristics of the research subjects

\begin{tabular}{lccc}
\hline \multicolumn{1}{c}{ Characteristics } & \multicolumn{2}{c}{ Groups } & \\
\cline { 2 - 4 } & Healthy individuals & Latent TB & 16 \\
\hline Number of subjects & 16 & 16 & Active TB \\
Sex $(\%)$ & & & $12(75 \%)$ \\
Male & $8(50 \%)$ & $5(31.3 \%)$ & $4(25 \%)$ \\
Female & $8(50 \%)$ & $11(68.8 \%)$ & $38.19 \pm 15.821$ \\
Age (Mean \pm SD) & $37.94 \pm 10.325$ & $39.88 \pm 11.008$ & $18-60$ \\
Age range & $18-53$ & $26-58$ & \\
\hline
\end{tabular}

ward, they were stored at $-70^{\circ} \mathrm{C}$ until IFN- $\gamma$, and IL10 levels were examined. The levels of IFN- $\psi$, and IL10 after the stimulation of the ESAT-6, and CFP-10 fusion antigens were then measured by using a sandwich method of Enzyme-Linked Immunoassay (ELISA) based on the procedures of the Human IFN- $\gamma$ and IL-10 U-CyTech Biosciences insert kits. After that, the differences in the mean IFN- $\gamma$ and IL-10 levels between those three groups after the stimulation of the ESAT- 6 and CFP-10 fusion antigens were analyzed by one-way ANOVA with a significance level of $\mathrm{p}<0.05$.

\section{RESULT AND DISCUSSION}

The quality control of IFN- $\gamma$, and IL-10 levels was performed with an impression control technique aimed to search for within-run impression by duplicating the examination on those ten samples
Unlike those two groups, the number of males in the healthy group was the same as the number of females, as many as eight individuals (50\%). In other words, males had a higher risk factor for active TB than females. Similarly according to the Global Tuberculosis Report in 2015, in 9.6 million new cases of TB in the world, 5.4 million of them were found in male patients, while 3.2 million of them were found in females, and 1 million of them were found in children. ${ }^{17}$

Furthermore, the mean age of the three groups was not much different (almost same) as depicted in Table 1.

The highest mean IFN- $\gamma$ level after the stimulation of ESAT-6, and CFP-10 antigens was found in healthy individuals. However, there was no significant difference in the mean IFN- $\gamma$ levels among those three groups ( $p=0.359$ ) as illustrated in Table 2 
Similarly, a research conducted by Surcel, et al., ${ }^{18}$ showed that there was no significant difference in IFN- $p$ produced by lymphocytes in PBMC of TB patients and healthy individuals who received in vitro M.tuberculosis antigen stimulation $(p>0.05) .{ }^{18}$ Increased proinflammatory cytokine production by ESAT-6, and CFP-10 fusion antigens then may increase antigen benefit for vaccine development. ${ }^{9}$ dietary protein (20\%). Those rats with low-protein diets indicated the decreased levels of IFN- $\gamma$, TNF- $\alpha$, and nitric oxide. ${ }^{20}$

The mean levels of IL-10 after the stimulation of ESAT 6, and CFP-10 antigens in active TB patients, latent TB patients, and healthy individuals increased. IL -10 levels in PBMC of healthy individuals were higher than in latent TB, and active TB patients, ranging from

Table 2. IFN- $\gamma$ levels in PBMC of patients with active and latent TB as well as healthy individuals after the stimulation of ESAT-6, and CFP-10 antigens

\begin{tabular}{lcccc}
\hline & & Groups & & \multirow{2}{*}{ p-value* } \\
\cline { 2 - 4 } IFN- $\boldsymbol{\nu}$ Levels & Active TB & Latent TB & Healthy & 0.359 \\
\hline Mean \pm SD & $371.38 \pm 721.28$ & $260.12 \pm 368.56$ & $359.17 \pm 526.40$ & \\
Median (min-max) & $27.39(0.07-2114)$ & $115.53(6.84-1381)$ & 180.09 & $(1.88-1807.70)$ \\
& & & & \\
\hline
\end{tabular}

* Significant if $p<0.05$

In this research, IFN- $\gamma$ levels in latent TB patients after stimulation of ESAT-6, and CFP-10 antigens were lower than in healthy individuals. Nevertheless, factors causing the lower IFN levels in those
31.30 to $958 \mathrm{pg} / \mathrm{mL}$ with a mean value of $215.37 \mathrm{pg} /$ $\mathrm{mL}$, and a standard deviation of $218.75 \mathrm{pg} / \mathrm{mL}$. However, there was no significant difference in the levels of IL-10 among the three groups after the stimulation of

Table 3. IL-10 levels in PBMC of patients with active and latent TB as well as healthy individuals after the stimulation of ESAT-6, and CFP-10 antigens

\begin{tabular}{ccccc}
\hline & & Groups & Healthy & p-value* \\
\cline { 2 - 4 } IL-10 & Active TB & Latent TB & & \\
\hline Mean \pm SD & $177.71 \pm 109.36$ & $175.80 \pm 100.15$ & $215.37 \pm 218.75$ & 0.712 \\
Median (min-max) & 181.30 & 170.25 & 170.10 & $(31.30-958)$ \\
\hline
\end{tabular}

* Significant if $p<0.05$

latent TB patients were not studied further in this research. There were some factors causing it, including the effects of genetic factors as, reviewed by Maderuelo et al., ${ }^{19}$ Maderuelo et al., ${ }^{19}$ argued that there was an abnormal IFN- $\gamma+874 \mathrm{~T} / \mathrm{A}$ gene polymorphism, playing a role in the production of IFN- $-{ }^{19}$

Besides, the status of host's nutrition may also affect the production of IFN- $\gamma$. Unfortunately, this research could not prove how much influence the nutritional status had on the immune response. Research conducted by Chandra ${ }^{20}$ ever found that nutrient deficiency can lead to decreased immune response, phagocyte function, cytokine production, and the complement system. This previous research showed how rats intentionally were given a lowprotein diet (2\%) were more susceptible to M.tuberculosis infection than rats getting enough
ESAT- 6 , and CFP-10 antigens $(p=0.712)$ as presented in Table 3.

As the results of this research, a research conducted by Joshi, et $a l^{21}$ also revealed that there was an elevated level of IL-10 in active, and latent TB serums compared to in healthy ones, but the difference was not significant. The higher mean IL-10 concentration in healthy individuals than in the other two groups is caused by polymorphism genes affecting the in-vitro secretion of cytokines. Similarly, there is an opinion that mutations in cytokine genes can affect the number of cytokines produced, resulting in an irregular immune response. ${ }^{21} \mathrm{IL}-10$ actually can also be produced by other immune cells, such as $\mathrm{TCD}^{+}$, B cells, eosinophils, and mast cells, but the pathways used by these cells to induce IL-10 are still not known. ${ }^{16}$ Besides, the increased levels of IL -10 can also be caused by other 
infections, especially infections that often occur in tropical countries. ${ }^{22}$

\section{CONCLUSION AND SUGGESTION}

In conclusion, there was no difference in IFN$\gamma$, and IL-10 levels of all three groups after simulation of M.tuberculosis antigens, ESAT-6, and CFP-10. Therefore, it is necessary for further researches to focus more on nutritional status and also to conduct in areas that are not endemic TB, so the possibility of exposure to M.tuberculosis is smaller than in this study.

\section{REFERENCES}

1. Kemenkes Republik Indonesia 2015. Profil Kesehatan Indonesia 2015. Available at http:// www.kemkes.go.id. Diunduh tanggal 1 Januari 2015

2. Ahmad S. Review: Pathogenesis, Immunology, and Diagnosis of Latent Mycobacterium tuberculosis Infection. Clinical, and Developmental Immunology. 2010; 2011: 1-17

3. Herrera V, Perry S, Parsonnet J, Banaei N. Clinical Application, and Limitations of Interferon- $\gamma$ Release Assays for the Diagnosis of Latent Tuberculosis Infection. Clinical Infectious Disease. 2011; 52(8): 1031-37

4. Keyser ED, Keyser FD, Baets FD. Tuberculin Skin Test versus Interferon-Gamma Release Assays for the Diagnosis of Tuberculosis Infection. Acta Clinica Belgia. 2014; 69(5): 358-67

5. Gyoung HY, Stolinska PG, Smith AB, Lalor MK, Chaguluka $\mathrm{S}$, et al. Combination of Cytokine Responses Indicative of Latent TB, and Active TB in Malawian Adults. Plos One. 2013; 8(11): 1-10

6. O'Garra A, Redford PS, McNab FW, Bloom Cl, Wilkinson RJ, et al. The Immune Response in Tuberculosis. The Annual Review of Immunology. 2013; 31: 475527

7. $\quad \mathrm{Xu} J \mathrm{~N}$, Chen JP, Chen DL. Serodiagnosis Efficacy and Immunogenicity of the Fusion Protein of Mycobacterium tuberculosis Composed of the 10-Kilodalton Culture Filtrate Protein, ESAT-6, and the Extracellular Domain Fragment of PPE68. Clinical, and Vaccine Immunology. 2012; 19(4): 536-544

8. Mustafa AS. Mini Review: Diagnostic and Vaccine Potentials of ESAT-6 Family Proteins Encoded by M. tuberculosis Genomic Regions Absent in M. bovis BCG. J Mycobacterial Diseases. 2013; 3(2): 1-5

9. Yuk JM, Jo EK. Review article: Host Immune Responses to Mycobacterial Antigens and Their Implications for the Development of Vaccine to Control Tuberculosis. Clinical Experimental Vaccine Research. 2014; 4(3): 155-167
10. Nikokar I, Makvandi M, Kajbaf MJ, Farajzadeh A, Mirsaeidi $\mathrm{M}$, et al. Evaluation of Cellular Immune Respons Against Purified Antigen 85 in Patients with Tuberculosis. Iran Journal Immunology. 2006; 3(2): 70-77

11. Fillon LG, Al-Ahdal MN, Tetro JA. Review: Vaccines against Mycobacterium tuberculosis : Exploring Alternate Strategies to Combat a Near-perfect Pathogen. J Mycobacterial Disease. 2013; 1-9

12. Cooper AM, Dalton DK, Stewart TA, Griffin JP, Russell DG, and Orme IM. Disseminated tuberculosis in interferon $\gamma$ gene-disrupted mice, Journal of Experimental Medicine, 1993; 178(6): 2243-47

13. Flynn JL, Chan J, Triebold KJ, Dalton DK, Stewart TA, and Bloom BR. An essential role for interferon $\gamma$ in resistance to Mycobacterium tuberculosis infection, Journal of Experimental Medicine, 1993; 178 (6): 2249-2254

14. Cavalcanti YVN, Brelaz MCA, Neves JK, Ferraz JC, Pereira VRA. Review: Role of TNF-Alpha, IFN-Gamma, and IL-10 in the Development of Pulmonary Tuberculosis. Hindawi Publishing Corporation. Pulmonary Medicine. 2012; 2012: 1-10.

15. Kassa D, Ran L, Geberemeskel W, Tebeje M, Alemu A, et al. Analysis of Immune Responses against a Wide Range of Mycobacterium tuberculosis Antigens in Patients with Active Pulmonary Tuberculosis. Clinical, and Vaccine Immunology, 2012; 19(12): 1907-1915.

16. Redford PS, Murray PJ, O'Garra A. The Role of IL-10 in Immune Regulation During M.tuberculosis Infection. Mucosal Immunology. Nature Publishing Group. 2011; 4 (3): 261-270.

17. WHO, 2015. Global Tuberculosis report 2015. WHO, Geneva, Switzerland.

18. http://www.who.int/tb/publications/global_report/en/. Diunduh tanggal 1 Januari 2015.

19. Surcel HM, Troye-Blomberg M, Paulline S. Th1/Th2 profiles in tuberculosis, based on proliferation, and cytokine response of blood lymphocytes to mycobacterial antigens. Immunology. 1994; 81(2): 171-176

20. Maderuelo DL, Arnalich F, Serantes R, Gonzales A, Codoceo R, Madero R. Interferon gamma, and interleukin-10 gene Polymorphisms in Pulmonary tuberculosis. Am J Respir Crit Care Med. 2003; 167: 970-975.Chandra RK. Nutrition, immunity, and infection : From basknowledge of dietary manipulation of immune responses to practical application of ameliorating suffering, and improving survival. Proc Natl Acad Sci USA. 1996; 93: 14304 - 7.

21. Joshi L, Ponnana $M$, Sivangala $R$, Chelluri LK, Nallari $P$, Penmetsa $S$, et al. Evaluation of TNF- $\alpha$, IL-10 and IL-6 cytokine production and their correlation with Genotype variants amongst Tuberculosis patients and their Household Contacts. Plos one, 2015; 10(9): 1-15. Doi: 10.1371/journal.pone.0137727.

22. Olobo JO, Geletu M, Demissie A, Eguale T, Hiwot K, Aderaye G, Britton S.Circulating TNF-a, TGFß, and IL-10 